\title{
La gestion durable des forêts tropicales. De l'analyse critique du concept à l'évaluation environnementale des dispositifs de gestion
}

Analyse réalisée par :

Christophe VoreuX

AgroParisTech Nancy

Corédacteur en chef de la Revue forestière française

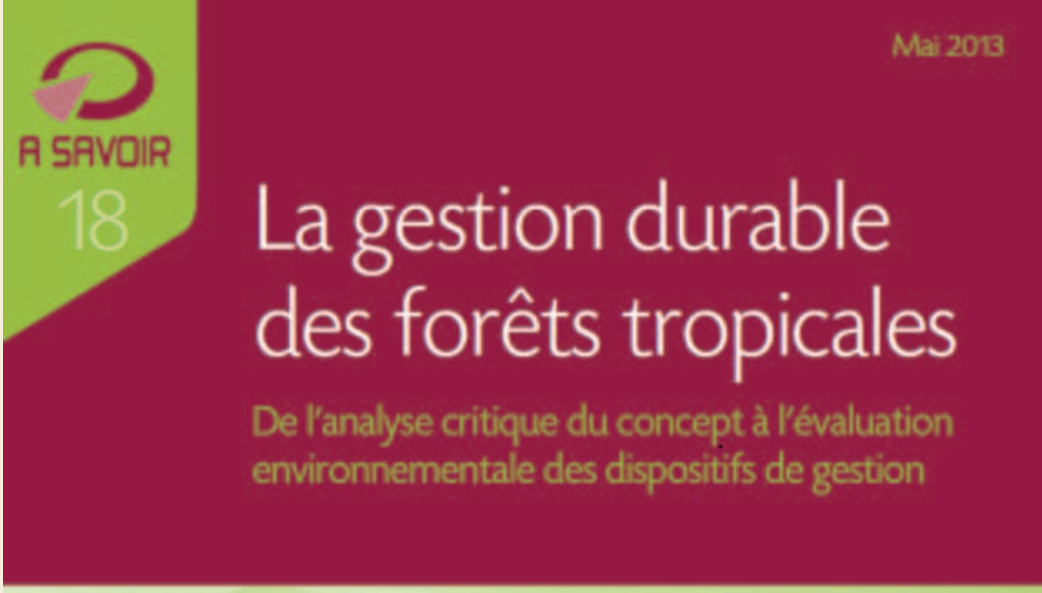

Maya LEROY

AgroParisTech

Géraldine DERROIRE

AgroParisTech

Jeremy VENDE

AgroParisTech

Tiphaine LEMENAGER

AFt

\section{Leroy, G. Derroire,}

J. Vendé, T. Leménager

FRANCE, AGENCE FRANÇAISE DE DÉVELOPPEMENT, COLLECTION « À SAVOIR », $\mathrm{N}^{0}$ 18, MAI 2013. $236 \mathrm{P}$.

Disponible gratuitement en téléchargement sur www.afd.fr 
Depuis 1990, la gestion durable des forêts (GDF) s'est progressivement instituée comme le cadre conceptuel en matière de gestion des forêts. Elle est souvent présentée, notamment en zone tropicale, comme seule capable de répondre efficacement à l'urgence environnementale actuelle. Toutefois, peu de travaux ont cherché à dresser un bilan de la façon dont la GDF a effectivement pris en charge les enjeux environnementaux par les multiples dispositifs mis en œuvre depuis plus de deux décennies. L'état actuel des forêts tropicales, ainsi que le rythme - qualifié par la FAO d' " alarmant » - de leur déforestation et de leur dégradation, fondent pourtant la légitimité de cette étude.

Sur la base d'une approche théorique en sciences de gestion, d'une méthodologie rigoureuse s'appuyant sur des analyses bibliographiques et lexicométriques de plus de deux mille cinq cents références et sur une quarantaine d'entretiens semi-directifs menés auprès d'acteurs clés, cet ouvrage constitue une analyse éclairante et originale sur la GDF et sa prise en charge des enjeux environnementaux dans les forêts tropicales.

Le public visé par ce livre n'étant pas forcément forestier, les auteurs ont bien pris soin de définir leur méthode de travail ainsi que certaines notions comme la sylviculture, le rendement soutenu, l'aménagement forestier. On regrettera toutefois que les explications apportées soient parfois assorties de commentaires peu objectifs, tel celui-ci :

"Cette démarche [la recherche du rendement soutenu] soutient donc le fait que les écosystèmes forestiers peuvent être gérés comme n'importe quelle autre entreprise industrielle, et que la nature peut être modelée pour s'adapter au paradigme d'industrialisation et de commercialisation. »

N'importe quelle autre ! C'est vraiment exagéré.

Le concept de gestion durable des forêts s'est répandu en même temps que celui de développement durable au début des années 1990. Trois définitions majeures, mais différentes, de la gestion durable des forêts sont citées, et il est peut-être utile de les mentionner ici.

Pour l'Organisation internationale des bois tropicaux, en 1992, la gestion durable des forêts, c'est

« la gestion de forêts permanentes en vue d'objectifs clairement définis concernant la production soutenue de biens et services désirés sans porter atteinte à leur valeur intrinsèque ni compromettre leur productivité future et sans susciter d'effets indésirables sur l'environnement physique et social ».

Les Nations unies ont, quant à elles, adopté à Rio de Janeiro, en 1992, la déclaration suivante, faisant autorité mais non juridiquement contraignante :

" Les ressources forestières et les terrains boisés devraient être gérés sur une base durable afin de répondre aux besoins sociaux, économiques, écologiques, culturels, spirituels, des générations actuelles et futures. Ces besoins concernent les produits et services que peut fournir la forêt tels que le bois et les produits à base de bois, l'eau, de la nourriture, du fourrage, des produits médicinaux, des combustibles, un abri, la création d'emplois, un lieu de détente, un habitat pour la faune sauvage, une source de diversité dans le paysage, le rôle de puits et de réservoir de carbone et bien d'autres produits tirés de la forêt. Des mesures appropriées devraient être prises en vue de protéger les forêts contre les effets dommageables de la pollution (et notamment de la pollution atmosphérique), des incendies, des insectes ravageurs et des maladies, afin de conserver intégralement tout ce qui en fait le prix. »

Enfin, la définition sans doute la plus connue est celle adoptée par la Conférence ministérielle sur la protection des forêts en Europe, en 1993 :

"La gestion durable des forêts signifie la gestion et l'utilisation des forêts et des terrains boisés d'une manière et à une intensité telles qu'elles maintiennent leur diversité biologique, leur productivité, leur capacité de régénération, leur vitalité et leur capacité à satisfaire, actuellement et pour le futur, les fonctions écologiques, économiques et sociales pertinentes aux niveaux local, national et mondial, et qu'elles ne causent pas de préjudices à d'autres écosystèmes. »

La suite du livre se recentre sur les forêts tropicales. Héritant au départ des pratiques passées, le concept de gestion durable des forêts s'est peu à peu institutionnalisé d'un point de vue réglementaire, y compris dans les pays tropicaux, sous l'influence de négociations et d'instances internationales. La deuxième partie présente les différentes façons dont la GDF a été traduite en instruments juridiques (traités, codes, lois, décrets...) pendant et après une phase intense de débats internationaux sur les forêts et l'élaboration de plans forestiers nationaux. La base de données FAOLEX a été utilisée à cette fin : c'est une base de données législatives informatisée proposée par le bureau juridique de la FAO, complète et à jour, qui représente la plus importante collection électronique de lois et de règlements nationaux, ainsi que de traités internationaux, portant sur l'alimentation, l'agriculture et les ressources naturelles renouvelables. Cinquante-six pays ou régions (dont la Guyane) ont été soumis à l'analyse de leur corpus juridique forestier, avec cependant un travail moins poussé pour dix-sept pays en raison de l'insuffisante connaissance par les auteurs des langues utilisées. Il a été tenu compte des différents systèmes juridiques de l'aire étudiée droit codifié ; droit jurisprudentiel ou common law ; droit musulman ; droit coutumier ; bijuridisme -, ce qui nécessite une grande précaution. (Une erreur de traduction relevée p. 61 , ligne 15 : lire richesse et non santé.) Les faits majeurs qui ressortent sur 1987-2010 sont les suivants : la généralisation des aménagements forestiers, la sécurisation des conditions d'octroi de concessions, la promotion de la participation des citoyens, le soutien fiscal accru à l'exploitation et la meilleure définition des forêts hors production (aires naturelles protégées, forêts sacrées, etc.). Une meilleure application des outils juridiques a aussi été en vue, en particulier dans les programmes de lutte contre le commerce du bois récolté illégalement.

Parallèlement à ce travail réglementaire, le concept de GDF a peu à peu été traduit en divers dispositifs de gestion qui, aujourd'hui encore, sont en évolution. En sciences de la gestion, on appelle dispositif de gestion un assemblage de règles de coordination (donc de jeux d'acteurs) et d'outils de gestion (donc de formalités techniques). La troisième partie aborde les dispositifs de gestion déployés au titre de la GDF dans les mêmes cinquante-six pays tropicaux. Trois catégories d'enjeux sont utilisées par les auteurs dans leur analyse de l'existant : $1^{\circ}$ améliorer l'exploitation forestière, avec 
l'aménagement forestier, la certification forestière et l'exploitation à faible impact ; $2^{\circ}$ valoriser le carbone stocké en forêt, avec les crédits carbone, volontaires ou reliés au protocole de Kyoto, et REDD+ ; $3^{\circ}$ accroître la participation des populations locales, avec la gestion conjointe des forêts, la foresterie communautaire, les forêts communales et encore d'autres formes de gestion participative. Dans tout cela, on relève que l'État se borne à assurer l'encadrement juridique des dispositifs qu'il promeut, mais que son rôle d'appui technique s'est effacé : les activités se déroulent souvent dans un cadre contractuel entre partenaires. La conclusion de cette partie, un peu difficile à suivre dans son exposé resserré, souligne que les dispositifs de gestion ont tendance à être utilisés concomitamment plutôt que seuls (à s'hybrider) par les acteurs de la gestion des forêts tropicales : ceci ne semble pas anormal dans la mesure où la GDF vise essentiellement à satisfaire simultanément des attentes variées des hommes vis-à-vis des forêts.

La quatrième partie traite de la prise en compte de l'environnement dans la GDF. Le corpus analysé par lexicométrie consiste en 1361 textes scientifiques sur ce sujet. L'intérêt est d'abord majoritairement porté sur la déforestation dans les études. Celle-ci cède la place vers 1994 à la biodiversité, qui elle-même commence à s'effacer à partir de 2002 devant le changement climatique. (Ce résultat intéressant d'un changement du thème dominant au cours du temps aurait mérité d'être étayé numériquement.) Le rapprochement avec les résultats de la partie 3 indique que, contrairement au carbone, la biodiversité n'a pas bénéficié d'un dispositif de gestion pour sa promotion au sein de la GDF, alors même qu'au plan scientifique elle était au centre des études : cette asymétrie interroge.

L'évaluation des politiques forestières et des dispositifs de gestion qui se réclament de la GDF au regard de la conservation de la biodiversité est menée avec soin par les auteurs. Si quelques améliorations ont été constatées dans les outils juridiques forestiers des pays tropicaux, des critiques sont néanmoins formulées sur la capacité réelle de ces dispositifs juridiques à répondre effectivement aux enjeux environnementaux.

Les dispositifs de gestion constituent les principales réponses opérationnelles apportées par la GDF face à la crise environnementale forestière observée. Or c'est la dimension économique (et prioritairement, la rentabilité de l'exploitation forestière) qui demeure leur enjeu central. Ils sont globalement déployés en cherchant à intégrer les " contraintes » environnementales et sociales à l'économie du secteur forestier, et non l'inverse.

Il apparaît également que, dans la littérature, la GDF aborde la conservation de la biodiversité surtout en termes de conservation du couvert forestier et de certaines espèces commercialisables. Au fond, la biodiversité reste «l'oubliée » de la GDF, bien que les problèmes de biodiversité soient au cœur des enjeux des écosystèmes forestiers tropicaux, et qu'ils aient été mis en avant depuis plus de vingt ans.

Au plan social, l'analyse note le caractère fort incertain de l'apport de la gestion participative, avec des exemples très contrastés de réussites significatives et d'échecs manifestes.

Outre ces conclusions, il a été constaté qu'un nombre important de publications sont consacrées à l'évaluation environnementale et aux enjeux de sa mise en œuvre dans des régions aux écosystèmes remarquables, tels que les forêts tropicales. Toutefois, l'application de cette démarche aux activités forestières à proprement parler n'a pour l'instant suscité qu'un intérêt limité, ce qui laisse penser que les dispositifs de gestion déjà existants seraient considérés par les acteurs comme déjà durables, ne requérant pas la mise en œuvre d'une démarche évaluative environnementale. De plus, le problème est très souvent ramené à un problème de gouvernance du secteur forestier, et non à un problème de nature des dispositifs de gestion proposés ou de niveau de performance environnementale. Très peu de mesures sont donc prises pour vérifier que, en ce qui concerne ces enjeux, la gestion durable des forêts l'est en effet.

Pour conclure - la conclusion du livre en est en fait un très bon résumé -, les tensions semblent se maintenir à ce jour entre une gestion des forêts tropicales avant tout centrée sur l'exploitation forestière et un secteur de la conservation de la nature qui continue à être perçu comme pouvant bloquer le développement de cette filière. Dans un tel contexte, la prise en charge des enjeux environnementaux paraît traitée de façon marginale au regard des enjeux qui y sont attachés.

L'insuffisance des processus d'évaluation environnementale en matière de GDF est soulignée, et cela bien que certains outils de la GDF incluent le suivi d'indicateurs de gestion, mais de façon partielle et insuffisamment précise. Il existe pourtant deux moyens à la disposition des responsables forestiers : l'étude d'impact environnemental et l'évaluation environnementale stratégique. L'efficacité de la GDF pourrait aussi être améliorée par une meilleure articulation des connaissances du secteur forestier et du secteur de la conservation et par un dialogue intersectoriel systématique entre le secteur forestier et le reste des secteurs exerçant une pression sur les forêts (agriculture, mines, gaz, pétrole, infrastructures). Les auteurs estiment peu réaliste de croire que le secteur forestier tropical pourra à lui seul assurer une gestion forestière véritablement durable.

Au final, l'ouvrage est important par l'originalité du sujet traité et la qualité avec laquelle les auteurs mènent leur analyse et en exposent les fruits. Il stimule la réflexion en la nourrissant de constats documentés. Les étudiants forestiers de niveau master ou ingénieur, les chercheurs en sciences politiques, de l'homme et de la société, et surtout les responsables forestiers, politiques et associatifs en tireront tous profit. 